\title{
Polymicrobial Septic Shock with Multiorgan Dysfunction in an Otherwise Healthy Immunocompetent Patient
}

\author{
${ }^{1}$ Anuradha Makkar, ${ }^{2}$ Inam D Khan, ${ }^{3}$ KS Rajmohan, ${ }^{4}$ Syed A Hashmi, ${ }^{5}$ Lakshmi Nair, ${ }^{6}$ Alpana Gupta, ${ }^{7}$ Harleen Chopra,
}

${ }^{8}$ Priyanka Banerjee, ${ }^{9}$ Pragyan S Panda, ${ }^{10}$ Rajiv M Gupta

\begin{abstract}
Background: Sepsis is a significant cause of morbidity and mortality worldwide despite advanced critical life-support. Septic shock and multiorgan dysfunction is the terminal stage in critically ill patients leading to perfusion abnormalities, lactic acidosis, oliguria and altered mental status creating a rapid downhill course and mortality. Sepsis ensues through stages of exaggerated immune response including systemic inflammatory response syndrome, in the backdrop of infectious stimuli.

Case report: Polymicrobial septic shock with multiorgan dysfunction leads to demise in an otherwise healthy immunocompetent patient. Microbiological profile revealed Escherichia coli urinary tract infection (UTI), Staphylococcus sciuri bacteremia, Acinetobacter baumanii ventilator-associated pneumonia, and central line catheter tip Pseudomonas aeruginosa, thereby pointing towards polymicrobial sepsis. Neutropenia of 290/ $\mathrm{dL}$ along with serum procalcitonin $5 \mathrm{ng} / \mathrm{mL}$ was detected. An autopsy revealed anasarca, bilateral lung abscesses, Hepatosplenomegaly, intracerebral hemorrhage, consolidated lungs, myocardial hypertrophy, and acute tubular necrosis were observed. Bacterial colonies from lungs, liver and cerebrospinal fluid grew Pseudomonas aeruginosa.
\end{abstract}

Conclusion: Polymicrobial sepsis can rapidly deteriorate a patient and mandate aggressive fluid, inotrope, ventilation, and antimicrobial therapy, notwithstanding challenges in diagnosis, prognosis and optimal management.

Keywords: Atypical pneumonia, Multiorgan dysfunction, Polymicrobial, Sepsis, Septic shock.

How to cite this article: Makkar A, Khan ID, Rajmohan KS, Hashmi SA, Nair L, Gupta A, Chopra H, Banerjee P, Panda PS, Gupta RM. Polymicrobial Septic Shock with Multiorgan Dysfunction in an Otherwise Healthy Immunocompetent Patient. Journal of Medical Academics 2018;1(1):61-64.

\footnotetext{
${ }^{1}$ Professor and Head, ${ }^{2}$ Associate Professor, ${ }^{3}$ Senior Advisor and Head, ${ }^{4}$ Asst Professor, ${ }^{5,6,8,9}$ Assistant Professor, ${ }^{7}$ Senior Resident, ${ }^{10}$ Consultant and Professor

1,2,7-9 Department of Microbiology, Army College of Medical Sciences and Base Hospital, New Delhi, India

${ }^{3-6}$ Department of Pathology, Army College of Medical Sciences and Base Hospital, New Delhi, India

${ }^{10}$ Department of Microbiology, Army Hospital Research and Referral, New Delhi, India

Corresponding Author: Inam D Khan, Associate Professor Department of Microbiology, Army College of Medical Sciences and Base Hospital, New Delhi, India Mobile: +918076324060, e-mail: titan_afmc@yahoo.com,
}

\section{Source of support: Nil}

Conflict of interest: None

\section{INTRODUCTION}

Sepsis is a major cause of morbidity and mortality worldwide despite advanced critical life-support. It is the $11^{\text {th }}$ leading cause of death overall with attributable mortality to sepsis and severe sepsis being 30 to $50 \%$ and 50 to $60 \%$. Following a non-infectious or infectious insult, there is a preliminary systemic response which becomes overwhelming leading to systemic inflammatory response syndrome. When the compensatory anti-inflammatory reaction fails, it leads to immunomodulatory failure, severe sepsis, septic shock, and multiorgan dysfunction. Sepsis ensues prolonged hospital stay and aggressive exposure to a cocktail of antimicrobials thereby increasing healthcare costs and furthering antimicrobial resistance. $^{1-3}$

Septic shock and multiorgan dysfunction is the terminal stage in critically ill patients leading to perfusion abnormalities, lactic acidosis, oliguria and altered mental status creating a rapid downhill course and mortality. Sepsis ensues through stages of exaggerated immune response including systemic inflammatory response syndrome, in the backdrop of infectious stimuli. Polymicrobial septic shock with multiorgan dysfunction in an otherwise healthy immunocompetent patient is being presented. $^{4}$

\section{CASE REPORT}

A 46-year-old woman with no known history of chronic disease, presented with mild to moderate grade intermittent fever with chills, dry cough without hemoptysis, sore throat, malaise and generalized body aches of two weeks duration. Initial outpatient workup for tropical fever comprising malaria, typhoid, dengue, chikungunya was negative. Subsequently, the patient presented with a febrile toxic look, breathlessness at rest, tachycardia 134/ min, normal blood pressure with no postural variation and hypoxemia at room temperature requiring oxygen inhalation and was promptly admitted to acute care. Examination revealed posterior pharyngeal wall congestion with mucus plugs; bilateral harsh vesicular breath 
sounds with fine crackles without associated wheeze or chest pain. She was initiated on parenteral co-amoxiclav, amikacin, and clarithromycin while on oxygen support, bronchodilators through nebulization and supportive therapy. During her illness, her hemoglobin was 8.5 $\mathrm{mg} / \mathrm{dL}$, leucocytes $12800 / \mathrm{cu} \mathrm{mm}$ with neutrophils $86 \%$ and lymphocytes $07 \%$, platelets $77,000 / \mathrm{cu} \mathrm{mm}$, normal peripheral blood smear without atypical lymphocytes or toxic granules or malarial parasite. D-dimer was $9 \mathrm{mcg} /$ $\mathrm{mL}$ (normal $<0.5 \mathrm{mcg} / \mathrm{mL}$ ) with normal fibrinogen and co-agulogram. Renal function tests, serum electrolytes, and liver function tests were within normal limits except lactate dehydrogenase which was $555 \mathrm{IU} / \mathrm{mL}$. Urine was acidic with increased sugar, protein and leucocytes. Arterial blood gas, $\mathrm{pH}$ and oxygen saturation was maintained. Two-dimensional echocardiography revealed normal cardiac function with an ejection fraction of $65 \%$, normal valves, and pericardium. Ultrasonography abdomen revealed mild splenomegaly with minimal left-sided pleural effusion.

She deteriorated 12-hours post admission rapidly with worsening of breathlessness, hypoxemia on mask ventilation, impending type-1 respiratory failure, and hypotension. She was shifted to intensive care and initiated on mechanical ventilation and inotropes. Antimicrobials were changed to ticarcillin-clavulanate, teicoplanin, levofloxacin, artesunate, and oseltamivir along with methylprednisolone, central venous pressure guided fluids, stress ulcer prophylaxis, and lung protective ventilation. Influenza a seasonal, pandemic H1N1 and influenza B reverse transcriptase polymerase chain reaction, serology for epstein-barr virus (EBV), enterovirus and Hantavirus was negative. On 3rd day, she was detected to have myocardial dysfunction with secondary myocarditis with persistent sinus tachycardia for 48 hours, elevated natriuretic peptide, creatine-kinase muscle/brain and troponin-I, non-specific ST-T changes,

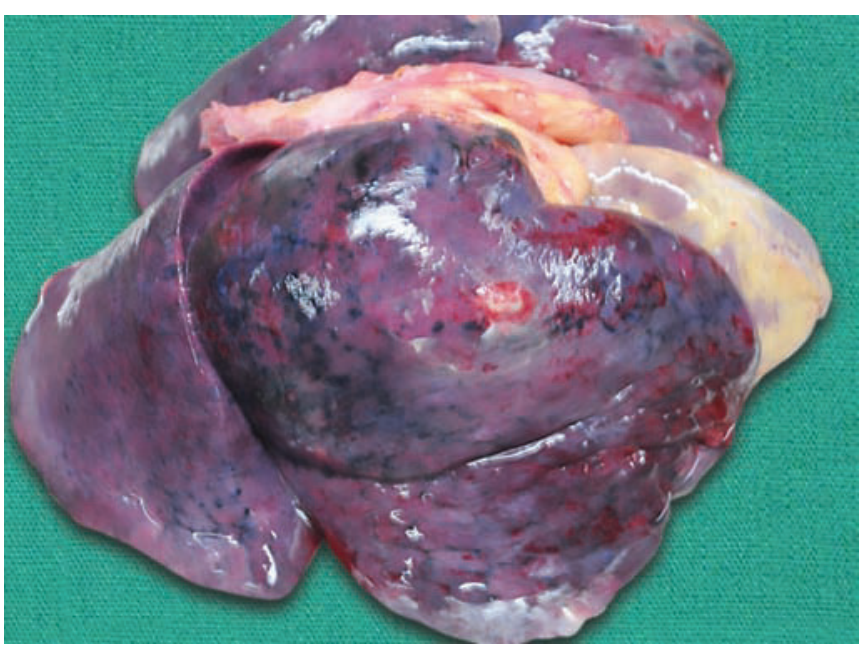

Fig 1: Lung abscess biventricular dysfunction on echocardiography, left and right ventricular ejection fraction $40 \%$ each. On 4 th day, she developed sudden bradycardia with refractory hypotension followed by an asystolic cardiac arrest for which she was resuscitated and continued on a ventilator and triple inotropic support while Glasgow Coma Scale (GCS) remained $E_{1} V_{T} M .1$ Further course was complicated by persistent high fever, stress hyperglycemia requiring insulin, refractory seizure requiring phenytoin, valproate, phenobarbitone and propofol, upper gastrointestinal bleed, hypernatremia, a deranged liver function which was managed conservatively. Iatrogenic pneumothorax and azotemia were managed by intercostal tube and peritoneal dialysis. Microbiological profile revealed Escherichia coli UTI, Staphylococcus sciuri bacteremia, Acinetobacter baumanii ventilator-associated pneumonia, and central line catheter tip Pseudomonas aeruginosa, thereby pointing towards polymicrobial sepsis. Neutropenia of 290/dl along with serum procalcitonin $5 \mathrm{ng} / \mathrm{mL}$ was detected. She sustained cardiac arrest on 14th day from which she couldn't be revived.

An autopsy revealed anasarca, ascites, bilateral lung abscesses, multiple petechiae over the heart and left kidney, congested greasy hepatosplenomegaly, and intracerebral hemorrhage in right basal ganglia (Fig. 1). Microscopically, pus from lung abscess showed Gram-negative bacilli. Congested and consolidated lungs, myocardial hypertrophy with a mononuclear interstitial infiltrate, macrovesicular liver steatosis and early chronic passive congestion, congested and dilated splenic red pulp with attenuated white pulp and acute tubular necrosis were observed microscopically. Bacterial colonies in the tissue spaces of lungs and liver as well as cerebrospinal fluid grew Pseudomonas aeruginosa which showed similar antibiogram typing profile as ante-mortem Pseudomonas (Figs 2 to 4 ).

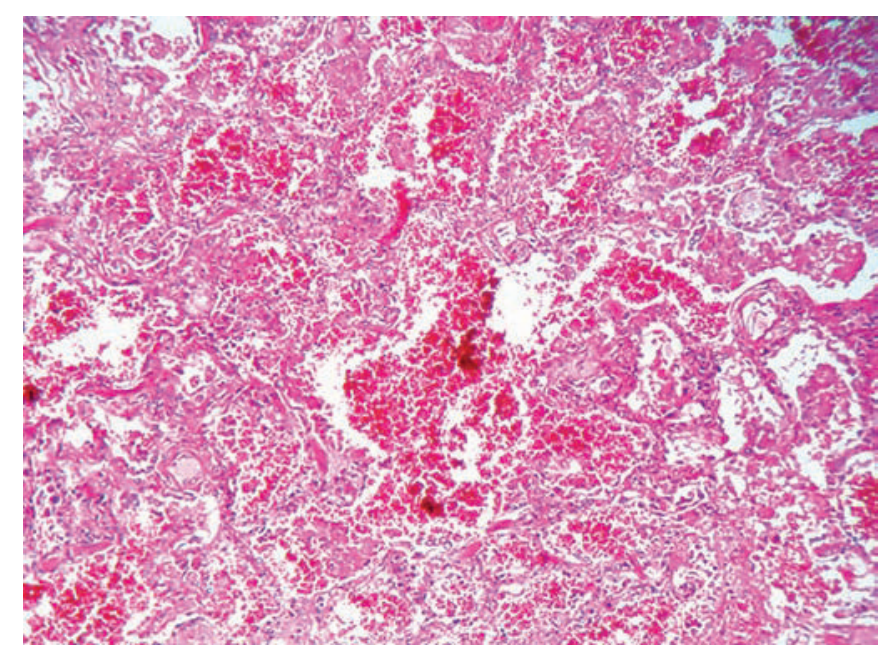

Fig 2: Congested and consolidated lung 


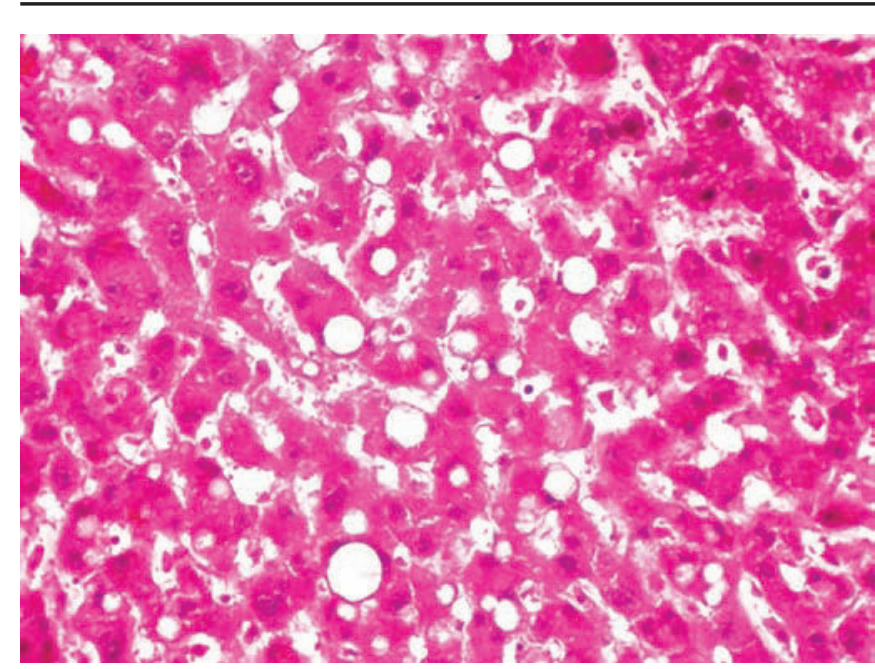

Fig 3: Macrovesicular steatosis of liver

\section{DISCUSSION}

Polymicrobial sepsis in this patient was evident from fever, tachypnoea, tachycardia, hypotension, $\mathrm{PaO}_{2} / \mathrm{FiO}_{2}$ $<250$, metabolic acidosis, azotemia, anemia, leucocytosis, thrombocytopenia, biventricular dysfunction requiring inotropic support and ventilator support. Polymicrobial sepsis with co-infective emerging bacterial and viral pathogens can cause severe disease which is worsened by healthcare-associated infections thereby obfuscating the clinical presentation and creating caveats to optimal diagnosis and treatment. ${ }^{5-10}$

Lipopolysaccharide, lipid A and endotoxin from Gram-negative pathogens, lipoteichoic acid from Grampositive pathogens, components from fungi, parasites and viruses stimulate. Toll-like receptor-mediated signaling leading to activation of NF- $\kappa \beta$ within monocytes. Proinflammatory cytokines, tumor necrosis factor- $\alpha$ (TNF- $\alpha$ ) and interleukine-1 released in bloodstream lead to the production of toxic downstream mediators, including prostaglandins, leukotrienes, platelet-activating factor, and phospholipase A2. These cytokines damage the endothelium and facilitate production of adhesion molecules on endothelial cells and neutrophils. Neutrophilendothelial interaction releases endothelium damaging neutrophil components and nitric oxide which is a potent vasodilator. $^{11}$

Interleukine-1 (IL-1) and TNF- $\alpha$ induce tissue factor expression on endothelium and monocytes leading to the production of thrombin resulting in fibrin clots in the microvasculature and impaired fibrinolysis due to the production of plasminogen activator inhibitor-1. Proinflammatory cytokines disrupt naturally occurring coagulatory and inflammatory mediators, activated protein C (APC) and antithrombin, leading to inhibition of factors Va and VIIIa, leukocyte adhesion and rolling, and neutrophil accumulation. ${ }^{12}$

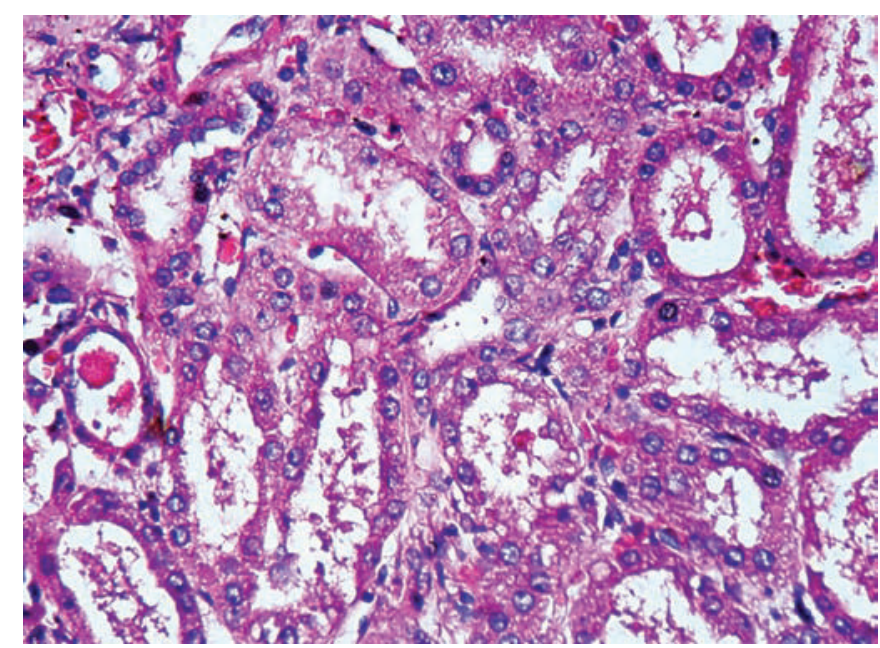

Fig 4: Acute tubular necrosis

CD4 lymphocytes initially assume a TH1 phenotype producing pro-inflammatory mediators including interferon gamma, TNF- $\alpha$, and IL-2, and later convert to Th2 phenotype producing anti-inflammatory cytokines including IL-10, IL-4 and IL-13 under the influence of stress hormones such as catecholamines and corticosteroids. These cytokines dampen the immune response, deactivate monocytes promote apoptosis of gut lymphocytes in the gut leading to immunoparalysis. Cardiovascular compromise occurs due to TNF induced myocardial depression, vasodilation, and capillary leak.

There are caveats to the approach to polymicrobial sepsis on clinical and diagnostic fronts. ${ }^{13}$ Biomarkers are heavily relied upon for diagnosis, prognosis, and initiation of antimicrobial therapy. Positive acute phase reactants such as $\mathrm{C}$-reactive proteins (CRP), procalcitonin, fibrinogen, serum amyloid A, factor B and D, mannosebinding protein, von Willebrand factor, haptoglobulin, C1-C9 complement components, $\alpha-1$ acid glycoprotein, $\alpha-1$ antitrypsin and properdin are increased, while albumin, transferring and antithrombin are reduced with ongoing infectious insult in sepsis. Various other cytokine/chemokines, cell markers, receptor markers, vasodilation markers, vascular endothelial damage markers, coagulation markers, organ dysfunction markers, apoptosis markers, selenium, saliva biomarkers, and emerging biomarkers are also under evaluation. CRP $>10 \mathrm{mg} / \mathrm{dL}$ reflects acute infection. Procalcitonin above $0.25 \mathrm{ng} / \mathrm{mL}$ is the cut off for initiation of antimicrobial therapy, between $0.5-2 \mathrm{ng} / \mathrm{mL}$ reflects sepsis, and $>2 \mathrm{ng} / \mathrm{ml}$ stratifies high risk of septic shock..$^{14,15}$

\section{CONCLUSION}

Polymicrobial sepsis can rapidly deteriorate a patient and mandate aggressive fluid, inotrope, ventilation, and antimicrobial therapy, notwithstanding challenges in 
diagnosis, prognosis and optimal management which demand intuitive mental mobility to discern role of infectious versus noninfectious insults, bacterial versus non-bacterial causation, antimicrobial initiation, escalation and de-escalation, diagnostic and prognostic correlates, pathophysiological compromise, drug toxicity and ongoing risk stratification.

\section{REFERENCES}

1. Jindal AK, Pandya K, Khan ID. Antimicrobial Resistance: A public health challenge. Med J Armed Forces India. 2014; 71 (2): 178-181.

2. Khan ID, Gupta RM, Sen S, Rajmohan KS, Jindal AK, Makkar A, et al. Emerging Antimicrobial Resistance and evolving healthcare: Dangerous crossroads for the community and the military. Journal of Archives in Military Medicine. In Press(In Press):e12097.

3. Khan ID, Rajmohan KS, Jindal AK, Gupta RM, Khan S, Shukla M, Singh S, Mustafa S, Tejus A, Narayanan S. Panresistant Superbugs: Are we at the edge of a "Microbial Holocaust". International Journal of Medicine and Medical Research. 2017; 3(2): 39-44.

4. Hotchkiss RS, Moldawer LL, Opal SM, Reinhart K, Turnbull IR, Vincent J-L. Sepsis and septic shock. Nature reviews Disease primers. 2016;2:16045.

5. Khan ID, Basu A, Kiran S, Trivedi S, Pandit P, Chattoraj A. Device-Associated Healthcare Associated Infections (DA$\mathrm{HAI}$ ) and the caveat of multiresistance in a multidisciplinary Intensive Care Unit. Med J Armed Forces India. 2017; 73 (3): 222-231.

6. Khan ID, Sahni AK, Bharadwaj R, Lall M, Jindal AK, Sashindran VK. Emerging Organisms in a Tertiary Healthcare Set Up. Med J Armed Forces India. 2014; 70 (2): 120-128.
7. Khan ID, Sahni AK. Bacterial Infections and Emerging Resistance in Renal Transplant Recipients.Bang J Med Sci. 2015; 14 (1): $14-21$.

8. Vijayvergia V, Sahni AK, Lal M, Vijay K, Khan ID. Phenotypic detection of ESBL and Amp C Beta-Lactamases in a tertiary care hospital. Bang J Med Sci. 2013; 12 (4): 378-384. www. banglajol.info/index.php/BJMS/article/download/13309/ 11724.

9. Khan ID, Lall M, Sen S, Ninawe SM, Chandola P. Multiresistant Elizabethkingia meningoseptica Infections in Tertiary Care. MJAFI 2014; 71 (3): 66-67.

10. Khan ID, Mukherjee T, Gupta S, Haleem S, Sahni AK, Banerjee S, Konar J. Ochrobactrum anthropi sepsis in intensive tertiary care. J Basic \& Clin Med 2014, 3(1): 18-20. http://sspublications.org/index.php/JBCM/article/view/35/32.

11. Iskander $\mathrm{KN}$, Osuchowski MF, Stearns-Kurosawa DJ, et al. Sepsis: Multiple Abnormalities, Heterogeneous Responses, and Evolving Understanding. Physiological Reviews.2013;93(3):1247-1288.

12. Singer M, Deutschman CS, Seymour CW, et al. The Third International Consensus Definitions for Sepsis and Septic Shock (Sepsis-3). JAMA. 2016;315(8):801-810.

13. Khan ID, Dogra PM, Ramphal SK, Khan S, Konar J, Palit A, Srivastava N, Agrawal P, Haleem S, Alam S. Polymicrobial infections in a teenaged Renal Transplant Recipient. J Basic \& Clin Med 2015, 4(1): 37-39. http://sspublications.org/index. $\mathrm{php/JBCM/article/view/41/39.}$

14. Khan ID, Sahni AK, Sen S, Gupta RM, Basu A. Outbreak of prototheca wickerhamii algaemia and sepsis in a tertiary care chemotherapy oncology unit. MJAFI 2017.

15. Reinhart K, Bauer M, Riedemann NC, Hartog CS. New Approaches to Sepsis: Molecular Diagnostics and Biomarkers. Clinical Microbiology Reviews. 2012;25(4):609-634. 